\section{Vanishing lung emphysema during chemotherapy for malignant pleural mesothelioma}

Ivana Castaniere, ${ }^{1}$ Roberto Tonelli, ${ }^{1}$ Sofia Taddei, ${ }^{2}$ Stefania Taschini, ${ }^{3}$ Riccardo Fantini, ${ }^{1}$

Alessandro Marchioni, ${ }^{1}$ Marisa Covi, ${ }^{1}$ Enrico M. Clini ${ }^{1}$

${ }^{1}$ Respiratory Diseases Unit, University Hospital of Modena, Modena;

${ }^{2}$ Respiratory Diseases Unit, Santa Maria Nuova Hospital, Reggio Emilia; ${ }^{3}$ Radiology Unit, University Hospital of Modena, Modena, Italy

\begin{abstract}
We report the case of a 79-year-old man with a tobacco smoke-related left dystrophic bullous emphysema that showed a considerable recovery of the cystic abnormalities during chemotherapy for pleural malignant mesothelioma. We suggest that the disappearance of the dystrophic emphysema could be explained by the combined effect of chemotherapy and pleural disease. We briefly review the literature and we discuss the possible mechanism of this unforeseen manifestation.
\end{abstract}

\section{Introduction}

Malignant pleural mesothelioma is a tumor that progressively involves the pleura growing circumferentially along the chest wall. As it arises from the pleura, different manifestations can occur, including pleural effusion, calcification and thickening, extension beyond the diaphragm with either contraction or enlargement of the hemithorax, mediastinum expansion and lymph nodes involvement. ${ }^{1-3}$ Herein, we describe an odd manifestation of mesothelioma occurring in a patient treated with first line chemotherapy. We have conducted a brief review of the literature and discussed the possible reasons for this unforeseen phenomenon.

\section{Case Report}

A 79-year-old man with asbestos exposure and a tobacco smoke-related dystrophic bullous emphysema - evident on chest Computed Tomography (CT) since he was 70 - presented in February 2011 with progressive shortness of breath and left chest pain. He underwent a chest CT scan that revealed left bullous parenchymal distortion associated to pleural thickening at the lower lobe and right pleural effusion (Figure 1). Considering the past environmental exposure, a transthoracic needle biopsy of the left pleura was performed and the diagnosis of malignant pleural mesothelioma was made. First line chemotherapy with cisplatin and pemetrexed was delayed for almost 3 months for the onset of infectious complications (pneumonia and urosepsis), then started with good toleration. Six weeks from treatment start (2 cycles given) another chest CT scan was performed showing a considerable reduction of the dystrophic cystic abnormalities with increased pleural thickness of the left lobe (Figure 2). During the time period between the two CT scan the patient experienced a significant improvement of the shortness of breath. Pulmonary function tests were performed showing an increased in lung volumes. (Forced Vital Capacity changed from 1,87 L to $2,13 \mathrm{~L}$ ). The lung pseudo-restoration remained evident in the $2 \mathrm{CT}$ scans performed until the patient died from septic shock 12 months later.

\section{Discussion and Conclusions}

With this brief report we present an unusual manifestation of pleural malignant mesothelioma during chemotherapy. A 79year-old man with dystrophic parenchymal bullae showed a consistent radiological regression of the lung emphysema during chemotherapy. Possible explanation for the disappearance of the dystrophic emphysema could be found in the rupture of the paraseptal bullae due to the treatment. ${ }^{4}$ There are very limited previously reported

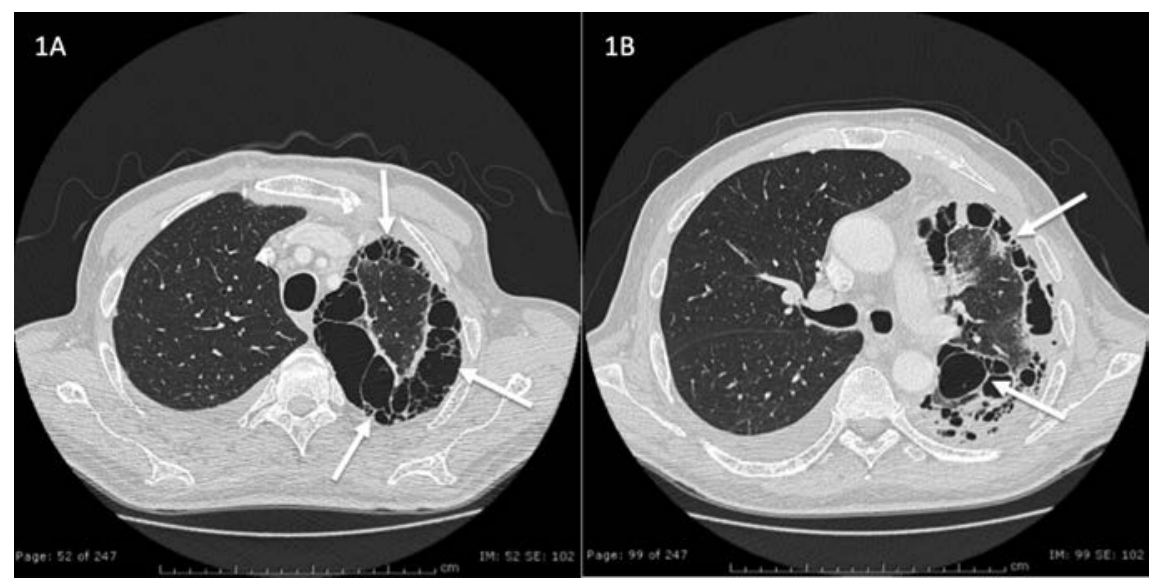

Figure 1. CT images of upper (A) and lower (B) lobes performed at the time of mesothelioma diagnosis showing left lung volume reduction with diffuse peripheral cystic-bullous abnormalities (white arrows), complete parenchymal distortion and right pleural effusion.

Correspondence: Roberto Tonelli, Respiratory Diseases Unit, University Hospital of Modena, Via del Pozzo 71, 41125 Modena, Italy.

Tel.: +39.059.4225086.

E-mail: roberto.tonelli@me.com

Key words: Mesothelioma; Emphysema.

Contributions: the authors contributed equally

Conflict of interest: the authors declare no potential conflict of interest.

Funding: none.

Informed consent: we state that have obtained written informed consent for publishing data from patient's relative.

Received for publication: 19 January 2018. Accepted for publication: 24 June 2018

This work is licensed under a Creative Commons Attribution NonCommercial 4.0 License (CC BY-NC 4.0).

C Copyright I. Castaniere et al., 2018

Licensee PAGEPress, Italy

Chest Disease Reports 2018; 6:7307

doi:10.4081/cdr:2018.7307

data on this phenomenon and most reports have been in patients with sarcoma or visceral pleural metastasis. ${ }^{5,6}$ Lee et al. described the onset of spontaneous pneumothorax resulting from intensive cytotoxic chemotherapy. ${ }^{6}$ Different pathogenic mechanisms have been hypothesized: in particular the tumor necrosis due to the cytotoxic effect of chemotherapy might directly induce a breach in emphysematous bullae. In our case pneumothorax did not occur, probably because of the symphysis due to pleural thickening; this could have fixed the

[Chest Disease Reports 2018; 6:7307]

[page 1] 


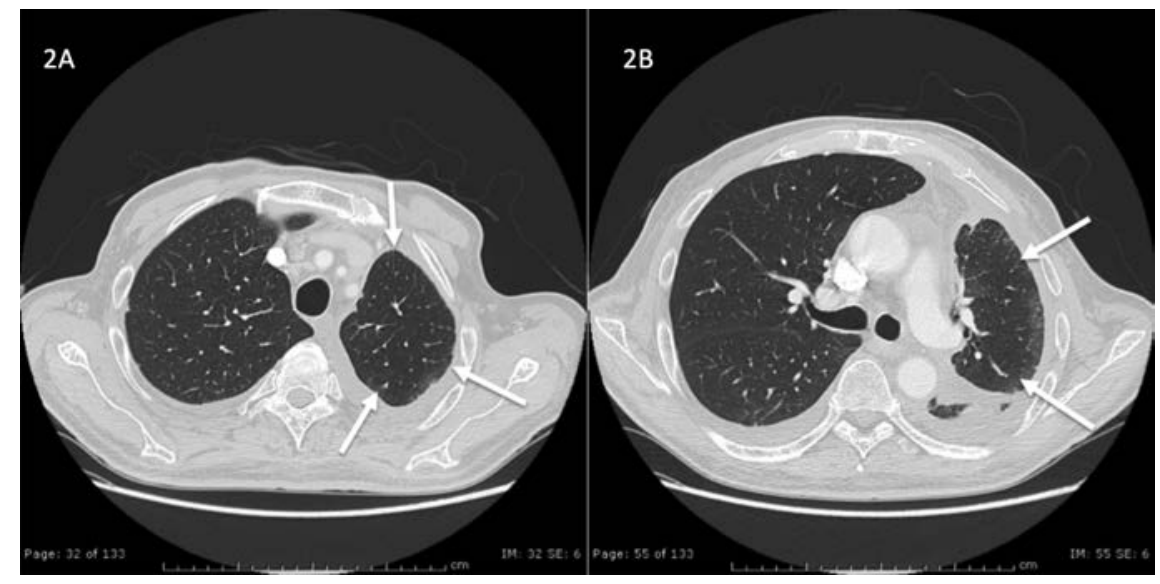

Figure 2. CT images of upper (A) and lower (B) lobes performed 3 months from chemotherapy start showing consistent reduction of the left pleural thickening with consensual disappearance of the dystrophic cysts and considerable regression of the parenchymal distortion at the left lower lobe (white arrows). Images show a mild increase of the right pleural effusion.

lung to the chest wall, avoiding its collapse and resulting in parenchymal re-expansion. While the effect of chemotherapy is one hypothesis, an alternative explanation may have been a circumferential growth of mesothelioma that engulfed the lung pulling the dystrophic emphysema towards the pleura. Thus the lung tissue stretching could have resulted in a pseudo-restoration. Even if this explanation might be acceptable, the chronological relationship with the beginning of treatment seems to reinforce the role of chemotherapy as the main cause of this phenomenon.

In conclusion, besides the unusual appearance, the depicted case seems interesting since it occurred in a patient undergoing chemotherapy. Through its description we aimed to present an unforeseen manifestation of pleural mesothelioma that has not been reported yet.

\section{References}

1. Au VW, Thomas M. Radiological manifestations of malignant pleural mesothelioma. Australas Radiol 2003; 47:111-6.

2. Ng CS, Munden RF, Libshitz HI. Malignant pleural mesothelioma: the spectrum of manifestations on CT in 70 cases. Clin Radiol 1999;54:415-21.

3. Fiorelli A, Vicidomini G, Napolitano F, Santini M. Spontaneous pneumothorax after chemotherapy for sarcoma with lung metastases: Case report and consideration of pathogenesis J Thorac Dis 2011;3:13840.

4. Jiang Hua, Ma Wei, Zhang Jing Ping, Zhang Lin. Spontaneous pneumothorax as the initial manifestation of stage I B primary pulmonary carcinoma: Really early stage cancer? J Canc Res Therapy 2013;9:118-20.

5. Nakano K, Motoi N, Tomomatsu J, et al. Risk factors for pneumothorax in advanced and/or metastatic soft tissue sarcoma patients during pazopanib treatment: a single-institute analysis. BMC Cancer 2016;16:750.

6. Lee CH, Park KU, Nah DY, Won KS. Spontaneous pneumothorax as a result of intensive cytotoxic chemotherapy: a case report. J Korean Med Sci 2003;18:227-80.

7. Hendarsih E, Fadjari TH, Oehadian A. Chemotherapy-induced spontaneous pneumothorax: Case series. Acta Med Indones 2016;48:134-8. 